\title{
Behaviour of microscale gas flows based on a power-law free path distribution function
}

\author{
Nishanth Dongari, Yonghao Zhang and Jason M Reese \\ Department of Mechanical Engineering, University of Strathclyde, Glasgow G1 1XJ, Scotland, United Kingdom \\ nishanth.dongari@strath.ac.uk
}

\begin{abstract}
We investigate a power-law form for the probability distribution function of free paths of dilute gas molecules in a confined region. A geometry-dependent effective molecular mean free path (MFP) model is then derived for a planar wall confinement, by taking into account the boundary limiting effects on the molecular paths. The power-law based effective MFP is validated against molecular dynamics simulation data and compared with exponential effective MFP models. The NavierStokes constitutive relations are then modified according to the kinetic theory of gases i.e. transport properties can be described in terms of the free paths which the molecules describe between collisions. Results for isothermal pressure-driven Poiseuille gas flows in micro-channels are reported, and we compare results with conventional hydrodynamic models, solutions of the Boltzmann equation and experimental data.
\end{abstract}

Keywords: Microchannel gas flow; Kndesn layer; Molecular mean free path; slip flows PACS: $47.61 .-\mathrm{k}, 47.61 . \mathrm{Cb}, 47.61 . \mathrm{Fg}$

\section{INTRODUCTION}

In microscale gas flows the small system dimensions mean that the non-dimensional Knudsen number, $K n$, defined as the ratio of the molecular mean free path (MFP) of the gas, $\lambda$, to a characteristic flow field dimension $(H)$, indicates the flows are often rarefied. As $K n$ increases, the behaviour of a gas flow near a solid bounding surface is dominated by the effect of gas molecule-surface interactions. This leads to the formation of a Knudsen layer (KL): a local thermodynamically non-equilibrium region extending $\sim O(\lambda)$ from the surface. Although the Navier-Stokes (NS) equations with classical velocity-slip and temperature-jump boundary conditions [1] can often adequately predict the flow field outside the KL, they fail to capture the non-linear form of the KL [2].

In the KL, gas molecule-surface collisions are more frequent than gas molecule-molecule collisions, i.e. the gas MFP will effectively be reduced in the KL. It is well-known from the kinetic theory of gases that viscosity and thermal conductivity can be interpreted in terms of the collisions of gas molecules, and of the free paths of the molecules between collisions [3]. Linear constitutive relations for shear stress and heat flux, with constant thermophysical transport coefficients such as viscosity and thermal conductivity, are not necessarily valid in the KL.

Stops [4] derived an effective MFP, $\lambda_{\operatorname{eff}(\mathrm{S})}$, which is geometry-dependent, by investigating the classical probability distribution function, $\psi(r)=\lambda^{-1} \exp (-r / \lambda)$. If the gas is not bounded, the MFP of the gas molecule ensemble is simply $\int_{0}^{\infty} r \psi(r) d r=\lambda$. If a solid bounding surface is included in the system, however, some molecules will hit the surface and their free flight paths will be terminated. The MFP of all the gas molecules in the system will therefore be smaller than $\lambda$, due to this boundary limiting effect. Guo et al. [6] implemented the effective MFP expression derived by Stops [4] in conjunction with a second-order slip boundary condition and obtained good results for pressure-driven rarefied gas flows.

However, comparison of these effective MFP profiles with molecular dynamics (MD) simulation data [7] shows that the model is accurate only up to Knudsen numbers of about 0.2 , as exponential distribution function can only provide an accurate description of a gas under thermodynamic equilibrium [4]. Non-equilibrium transport is often better described using Lévy and power-law (PL) probability distributions $[8,9]$.

This paper investigates the hypothesis that the transport behaviour of non-equilibrium gases is better understood by using PL type of distribution functions. We derive an effective MFP based on a PL probability distribution of free paths for a gas close to a planar wall, and show that this captures some of the trends associated with the complex nonequilibrium physics of transition regime gas flows. Our PL-based effective MFP is validated against MD simulation data [7] up to $K n=2$ and also compared with the theoretical models of Stops [4] and Arlemark et al. [5]. 


\section{AN EFFECTIVE MOLECULAR MEAN FREE PATH}

Let us consider a group of similar gas molecules moving at a speed $\bar{v}$ with a collision rate of $\dot{\theta}_{v}$, where molecular mean free path $\lambda=\bar{v} / \dot{\theta}_{v}$. The number in the group at time $t=0$ is $N_{0}$, and by time $t$ let $N$ of them still be travelling without having had a collision. During the interval $d t, N \dot{\theta}_{v} d t$ molecules will collide and drop out of the group; $N$ is thereby changed by the amount:

$$
d N=-N \dot{\theta}_{v} d t
$$

We can easily integrate this equation under thermodynamic equilibrium conditions, as the probability of a certain microscopic state averaged over the details of the interactions, does not change in time or space [3]. So, with $\dot{\theta}_{v}$ constant, $d N / N=-\dot{\theta}_{v} d t$, thus $\ln N=-\dot{\theta}_{v} t+$ const. Choosing the constant of integration so as to make $N=N_{0}$ at $t=$ 0 , we find

$$
N=N_{0} \exp \left(-\dot{\theta}_{v} t\right)=N_{0} \exp \left(-\frac{\dot{\theta}_{v}|r|}{\bar{v}}\right),
$$

where $|r|$ is the length $\bar{v} t$ of free path that has been travelled at time $t$ by each molecule. Let $\psi(r) d r$ be the fraction of all the free paths that have a length between $r$ and $r+d r$. Then $\psi(r) d r=d N / N_{0}$, hence from $\lambda=\frac{\bar{v}}{\dot{\theta}_{v}}$

$$
\psi(r)=\frac{1}{\lambda} \exp \left(-\frac{|r|}{\lambda}\right) .
$$

So $\psi(r)$, the probability distribution of free paths, is only exponential in form under thermodynamic equilibrium conditions and when the gas is unbounded.

Although it is certainly difficult to evaluate the probability distribution function $\psi(r)$ in Knudsen layer, introducing non-equilibrium into Eq. (1) by means of physical arguments could allow us to propose a distribution function that may be more appropriate for non-equilibrium conditions than the exponential one [3]. Montroll \& Scher [9] pointed out that a finite moment of the probability distribution function implies an exponential character of the randomness. A distribution function with diverging higher-order moments, such as the standard deviation, is essential to nonequilibrium transport.

We hypothesize a PL form for the probability distribution function for non-equilibrium MFP, with diverging higherorder moments, instead of the classical exponential form of distribution function. Here we propose for investigation the following form of molecular free path distribution function:

$$
\psi(r)=C(a+r)^{-n}
$$

where $a$ and $C$ are constants with positive values determined through the zero and first moments. The range of values for the exponent $n$ can be obtained by making one of the higher-order moments divergent. Zero and first moments are given as follows:

$$
\begin{gathered}
1=\int_{0}^{\infty} C(a+r)^{-n} d r, \\
\lambda=\int_{0}^{\infty} C r(a+r)^{-n} d r .
\end{gathered}
$$

Equation (5) requires the probability to range only from zero to one. Equation (6) defines the unconfined, conventional MFP value, $\lambda$. It then follows that, $C=(n-1) a^{n-1}$ and $a=\lambda(n-2)$. If $n>2, a>0$, otherwise the distribution function is negative. The standard deviation (second moment) of the distribution function given by Eq. (4) diverges only for $n \leq 3$; so $2<n \leq 3$. If one wishes to make the $i^{t h}$ moment diverging, then $\max (n)=i+1$. As $n \rightarrow \infty$, the distribution function will have finite moments, which is the condition required of an equilibrium distribution function. For a finite $n$, the distribution function describes a system deviating from equilibrium. Thus, $n$ acts as a decisive parameter to define the extent of deviation from equilibrium. Here, we test $n=3$, unless otherwise explicitly stated.

We follow the approach based on an integrated form of the probability distribution function [5], i.e.

$$
p(r)=\int_{r}^{\infty} \psi(r) d r=\left[1-\left(1+\frac{r}{a}\right)^{1-n}\right]
$$

which describes the probability a molecule travels a distance $r$ without experiencing a collision. Our model is derived for the two-planar-wall configuration. We use the notation $r^{-}$if a test molecule is travelling in the negative $y$-direction, 
and $r^{+}$if the molecule is travelling in the positive $y$-direction. We also use the notations $\theta^{-}$and $\theta^{+}$for the equally probable zenith angle travelling direction of the molecule. These quantities are related through $r^{-}=(H / 2+y) / \cos \theta^{-}$ and $r^{+}=(H / 2-y) / \cos \theta^{+}$, where $H$ is the distance between the two parallel plates.

The MFP based on the PL form of distribution function, $\lambda_{\text {eff(PL) }}$, is expressed by weighting the unconfined MFP, $\lambda$, with $p(r)$ as follows:

$$
\lambda_{\mathrm{eff}(\mathrm{PL})}=\frac{\lambda}{2}\left[p\left(r^{-}\right)+p\left(r^{+}\right)\right]=\lambda\left\{1-\frac{1}{2}\left[\left(1+\frac{r^{-}}{a}\right)^{1-n}+\left(1+\frac{r^{+}}{a}\right)^{1-n}\right]\right\} .
$$

A 3-dimensional MFP depending on the gas molecule's distance to a surface is then obtained by averaging the free path with respect to $\theta^{-}$and $\theta^{+}$in the range $[0, \pi / 2]$ using the mean integral theorem,

$$
<\lambda_{\mathrm{eff}(\mathrm{PL})}(\theta)>=\frac{2}{\pi} \int_{0}^{\pi / 2} \lambda_{\mathrm{eff}(\mathrm{PL})}(\theta) d \theta
$$

Averaging over the free path in Eq. (9), using Simpson's numerical integration involving 16 subintervals, results in $\lambda_{\mathrm{eff}(\mathrm{PL})}=\lambda \beta_{\mathrm{PL}}$, where

$$
\begin{aligned}
& \beta_{\mathrm{PL}}=1-\frac{1}{94}\left[\left(1+\frac{H / 2-y}{a}\right)^{1-n}+\left(1+\frac{H / 2+y}{a}\right)^{1-n}+4 \sum_{i=1}^{8}\left(1+\frac{H / 2-y}{a \cos [(2 i-1) \pi / 32]}\right)^{1-n}+4 \sum_{i=1}^{8}\left(1+\frac{H / 2+y}{a \cos [(2 i-1) \pi / 32]}\right)^{1-n}\right. \\
& \left.+2 \sum_{i=1}^{7}\left(1+\frac{H / 2-y}{a \cos [i \pi / 16]}\right)^{1-n}+2 \sum_{i=1}^{7}\left(1+\frac{H / 2+y}{a \cos [i \pi / 16]}\right)^{1-n}\right],
\end{aligned}
$$

which is the normalized effective MFP based on the power-law distribution function, and is evaluated using the rarefaction parameter $K n$ (as $a$ is dependent on mean free path).

\section{Comparison with Molecular Dynamics simulations}

The $\beta$-function of Eq. (10) is now compared with MD simulation results [7] and the classical exponential probability function models of Stops [4] and Arlemark et al. [5], for both single- and parallel-wall cases. Single-wall results are deduced by assuming that the second wall is located an infinite distance from the first.

Figure 1(a) shows the variation of normalized effective MFP profiles (i.e. $\beta$ ). Our PL model predictions are in close agreement with MD simulations, although minor overpredictions are noticed in the bulk region. The PL model has a sharp gradient close to the wall, while both of the previous exponential-based models have shallower gradients and underpredict the MFP values in the wall vicinity. All three theoretical models converge to a similar value in the bulk region as expected.

Results for the normalized MFP between two parallel surfaces are presented in Fig. 1(b). At $K n=0.2$, the PL model is in fair agreement with the MD data in the near-wall region and the two exponential based models underpredict in the KL. As the value of $K n$ increases, the classical models fail to predict the effective MFP in the wall region, as well as in the bulk. Both classical models underpredict the effective MFP values, although the Arlemark et al. [5] results are slightly above Stops'. PL model MFP values compare very well to the MD data for both $K n=0.5$ and 1 , although it overpredicts for $K n=1$ in the bulk. By $K n=2$, the PL model shows significant deviations from the simulation data and overpredicts in the near-wall region, although there is fair agreement in the bulk region. The MD data shows a relatively sharp gradient of effective MFP in the near-wall region, compared to the theoretical predictions.

\section{ISOTHERMAL PRESSURE-DRIVEN RAREFIED GAS FLOWS}

We consider isothermal pressure-driven gas flow along a planar-wall channel with walls a distance $H$ apart as a test case. The flow is assumed to be hydrodynamically developed and two-dimensional, isothermal, laminar and steady. With these assumptions, the governing equation is:

$$
0=-\frac{\partial P}{\partial x}-\frac{\partial \tau}{\partial y}
$$




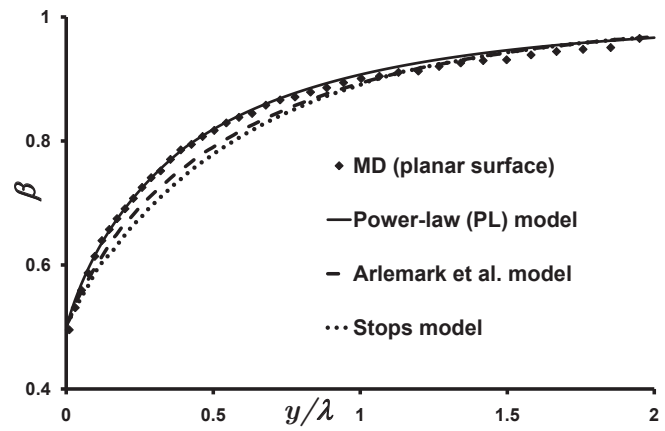

(a)

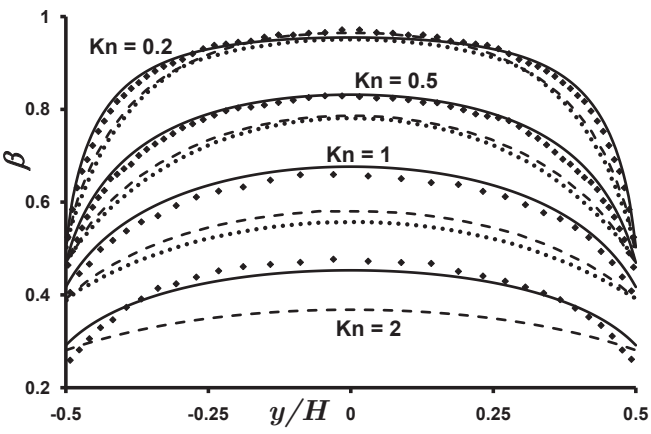

(b)

FIGURE 1. Variation of normalized mean free path, $\beta$, with normalized distance from a surface; (a) single-wall case and (b) parallel-wall case. Comparison of power-law (PL) model with molecular dynamics (MD) simulation data [7], and Arlemark et al. [5] and Stops [4] exponential models, for various Knudsen numbers.

where $x$ is the streamwise coordinate, $y$ the wall-normal coordinate, $P$ the pressure and $\tau$ the stress, which is given as:

$$
\tau=-\mu\left[\nabla \mathbf{U}+(\nabla \mathbf{U})^{t r}\right]+\left(\frac{2}{3} \mu-\kappa\right)(\nabla \cdot \mathbf{U}) I,
$$

where $\mu$ is the fluid dynamic viscosity, $\kappa$ the bulk viscosity, $I$ the identity tensor and $t r$ the transpose operator.

The unconfined MFP is related to the shear viscosity [3]:

$$
\mu=\rho \frac{\lambda}{\sqrt{\pi / 2 R T}}
$$

where $R$ is the specific gas constant and $T$ the gas temperature. Equation (13) is assumed to be valid only for flows that are quasi-equilibrium. If we wish to use Eq. (13), we need to take into account the MFP affected by gas molecular collisions with surfaces. If the unconfined expression for the MFP, $\lambda$, is replaced by our effective and geometrydependent mean free path, $\lambda_{\text {eff(PL) }}$, we obtain a non-constant, geometry-dependent, effective viscosity, $\mu_{\mathrm{eff}(\mathrm{PL})}$, that can then be used to postulate a non-linear stress/strain-rate relation:

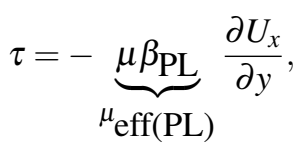

Using Eq. (14) in Eq. (11) results in the modified governing equation:

$$
\mu \frac{\partial}{\partial y}\left(\beta_{\mathrm{PL}} \frac{\partial U_{x}}{\partial y}\right)=\frac{\partial P}{\partial x}
$$

where $U_{x}$ is the fluid velocity in the axial direction. This needs to be solved in conjunction with an appropriate slip boundary condition. Guo et al. [6] proposed the following heuristic slip boundary condition to take into account the KL correction:

$$
U_{\text {slip }}=-C_{1}\left(\lambda_{\text {eff }} \frac{\partial U_{x}}{\partial y}\right)_{w}-C_{2}\left[\lambda_{\text {eff }} \frac{\partial}{\partial y}\left(\lambda_{\text {eff }} \frac{\partial U_{x}}{\partial y}\right)\right]_{w} .
$$

This simply reduces to the conventional second-order slip boundary condition [11] if there is no KL, i.e. if $\lambda_{\text {eff }}=$ const . Values of $C_{1}$ and $C_{2}$ are chosen as 1 and 0.31 [12] respectively for the results presented here.

The wall-normal coordinate $y$ is normalized by $H$, and the axial velocity $U_{x}$ by the free-molecular velocity $U_{0}=$ $-2 H(\partial P / \partial x) /(\rho \sqrt{2 R T})$ [13]. Resulting governing equation is numerically solved for the normalized axial velocity profiles $U^{*}$ by applying the slip boundary condition at the upper wall, $y^{*}=0.5$, and a symmetry condition at the centre of the channel, $y^{*}=0$. 

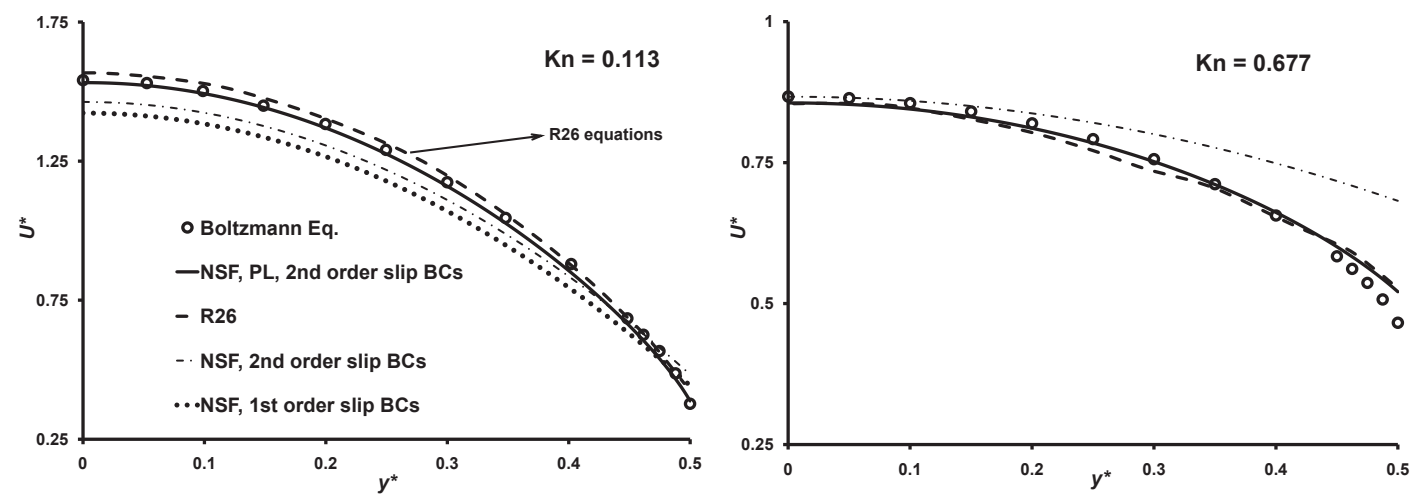

FIGURE 2. Normalized half-channel velocity profiles for various Knudsen numbers. Comparison of power-law (PL) model results with the solution of the Boltzmann equation [13], R26 moment equations (- -) [14] and conventional NS equations with first- and second-order slip (-.-).
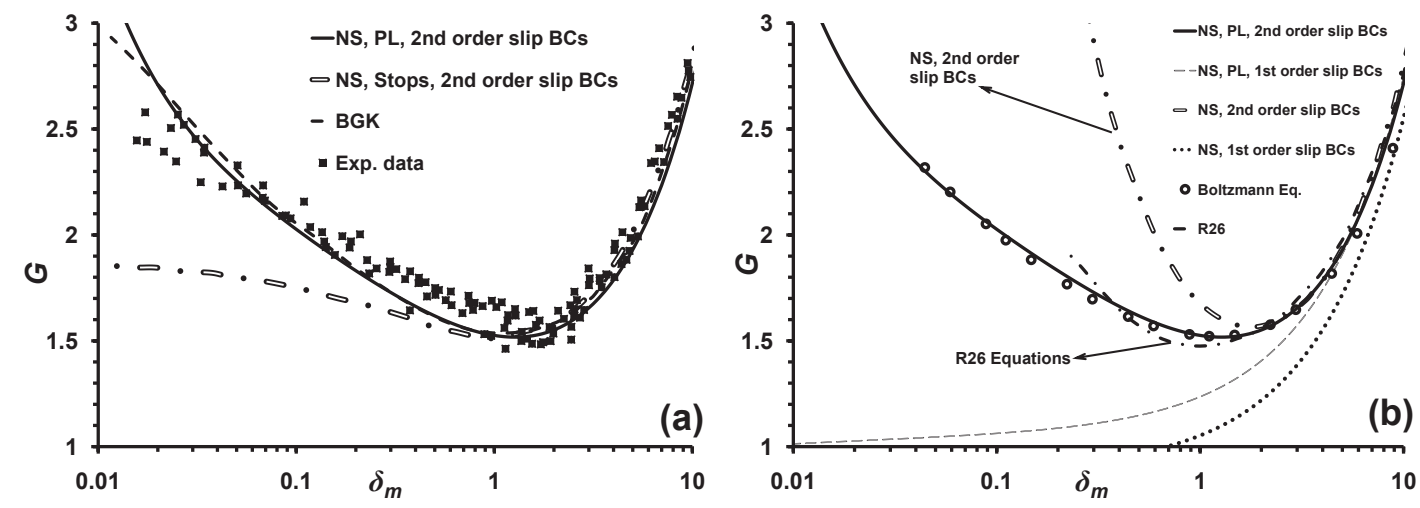

FIGURE 3. Normalized volume flow rate $(G)$ variation with inverse Knudsen number $\left(\delta_{m}\right)$. Comparison of power-law (PL) model results with: (a) experimental data [10] and BGK simulation results (--) [15]; (b) the solution of the Boltzmann equation [13], R26 equations (-.-) [14] and the conventional NS equations with first- and second-order slip.

\section{RESULTS AND DISCUSSION}

The cross-sectional velocity profiles of pressure-driven Poiseuille flow at a range of Knudsen numbers are presented in Fig. 2. Our PL model is validated with a Boltzmann solution [13] and compared with three hydrodynamic models: conventional first- and second-order slip models, and the R26 equations [14]. At $K n=0.113$, the PL model and R-26 equations predict similar values of slip velocity at the wall and are close to the solution obtained from the Boltzmann equation [13]. The conventional NS equations with both first- and second-order slip boundary conditions significantly underpredict the velocity in the bulk region and show minor deviations in the KL. The PL model is in fair agreement with the Boltzmann solution in the bulk region, although the R26 equations slightly overpredict the maximum velocity. At higher $K n$, the flow enters the transition regime and the non-equilibrium regions from both parallel walls start to overlap each other. At $K n=0.677$, the slip model shows significant discrepancy in the Knudsen layer and both the PL model and R26 equations compare reasonably well with the Boltzmann solutions, although the R26 equations overpredict the wall-slip velocity.

It is evident from Figs. 1 and 2 that in the transition regime, non-equilibrium effects are no longer limited to the walladjacent layer, but prevail in the bulk flow due to the overlap of Knudsen layers. Hence, simply using a higher-order slip model with modified slip coefficients may result in good wall-slip velocity results but will not improve the overall predictive capabilities of the NS equations in the transition regime. The NS equations with an exponential-based MFP can provide good velocity profiles in the Knudsen layer up to the early transition regime. However, with our PL model we could influence the velocity profile at a specified Knudsen number through the exponent $n$. 
Figure 3 shows the variation of normalized flow rate $G$ with inverse Knudsen number, $\delta_{m}=\sqrt{\pi} /(2 K n)$. In Fig. 3(a), the PL model results are compared with experimental data [10], BGK results [15] and the NS equations with Stops' MFP model and second-order slip condition. In the slip flow regime, both the PL model and Stops' MFP based slip model agree reasonably well with the experimental data and BGK simulation results. Around $\delta_{m} \sim 1$, Stops' MFP based slip model starts to significantly underpredict the flow rate, whilst the PL model follows the BGK simulation data reasonably well until $K n$ reaches about 10. Flow rate results of our PL model with $n=3$ are shown in Fig. 3(b) to compare with the exact Boltzmann solution [13], R26 results [14], NS equations with first- and second-order slip and the PL model with first-order slip. Here the first-order slip for the PL based MFP NS equations results are obtained simply by setting $C_{2}=0$. For $K n>0.1$, NS equations with first-order slip underpredict the flow rate, although the other hydrodynamic models are in good agreement with the Boltzmann solution up to $K n \sim 0.2$. The NS results with second-order slip are good up to $K n \sim 0.5$, but significantly overpredict beyond that. The R26 equations show good agreement until $K n \sim 2$, and our PL model with second-order slip follows the Boltzmann results up to $K n \sim 10$.

\section{CONCLUSIONS}

The key physics of the rarefied gas flow in the Knudsen layer has been outlined, and we have proposed an analysis based on a power-law (PL) probability distribution function for free-paths of molecules in a confined gas. Theoretical comparisons with the MD simulation data illustrates that exponential distribution functions are inaccurate in the transition regime and the PL model can predict non linear phenomenon in the Knudsen layer. A constitutive scaling approach to model the Knudsen layer within a continuum fluid dynamics framework has also been described. This has been tested for the case of isothermal pressure-driven gas flow through a microchannel. An advantage of the PL MFP constitutive scaling technique is that it is based on simple physical arguments, which can successfully be implemented in conventional CFD codes, and is still able to capture some of the essential trends associated with the complex non-equilibrium physics of the Knudsen layer.

While it is important not to draw strong conclusions based on these simple test cases alone, the present results may motivate future work into understanding the origin of non-equilibrium physics in rarefied gases, incluing:

1. validation of the power-law probability distribution function for a range of flow systems geometries through molecular dynamics simulations;

2. classical constitutive relationships and velocity slip boundary conditions are derived under the assumption of constant MFP in Knudsen layers - derivations of these boundary conditions should be revisited based on geometrydependent effective MFP models through an appropriate kinetic equation.

\section{ACKNOWLEDGMENTS}

The research leading to these results has received funding from the European Community's Seventh Framework Programme FP7/2007-2013 under grant agreement ITN GASMEMS no. 215504.

\section{REFERENCES}

1. J. C. Maxwell, Philosophical Transactions of Royal Society, Part 1, 170, 231-256 (1879).

2. D. A. Lockerby, J. M. Reese, and Gallis, M. A., Physics of Fluids, 17, 100609 (2005).

3. C. Cercignani, Mathematical Methods in Kinetic Theory, Plenum Press, New York, 1990.

4. D. W. Stops, Journal of Physics D: Applied Physics, 3, 685-696 (1970).

5. E. J. Arlemark, S. K. Dadzie, and J. M. Reese, Journal of Heat Transfer, 132, 041006 (2010).

6. Z. L. Guo, B. C. Shi, and C. G. Zheng, Europhysics Letter, 80 (2), 24001-24006 (2007).

7. E. J. Arlemark, and J. M. Reese, ASME ICNMM2009-82080, Pohang, South Korea (2009).

8. K. S. Fa, and E. K. Lenzi, Physical Review E, 67, 061105 (2003).

9. E. W. Montroll, and H. Scher, Journal of Statistical Physics, 9 (2), 101-135 (1973).

10. T. Ewart, P. Perrier, I. A. Graur and J. B. Meolans, Journal of Fluid Mechanics, 584, 337-356 (2007).

11. N. Dongari, A. Agrawal and A. Agrawal, International Journal of Heat Mass Transfer, 50, 3411-3421 (2007).

12. N. G. Hadjiconstantinou, Physics of Fluids, 15(8), 2352-2355 (2003).

13. T. Ohwada, Y. Sone, and K. Aoki, Physics of Fluids A 1, 2042-2049 (1989).

14. X. J. Gu, and D. R. Emerson, Journal of Fluid Mechanics, 636, 177-226 (2009).

15. S. K. Loyalka, Journal of Chemical Physics, 63, 4054-4560 (1975). 